\title{
11. Addiction sexuelle et couple
}

\author{
Philippe Kempeneers
}

Pour citer ce chapitre : Kempeneers, P. (2018). Addiction sexuelle et couple. In F. Allard \& P. Antoine (Eds.), Le couple en thérapie comportementale, cognitive et émotionnelle, pp. 239-256. Issy-les-Moulineaux : Elsevier Masson. ISBN: 978-2-294-75892-8.

\section{Qu'est-ce qu'une addiction ?}

De la manière la plus générale qui soit, une addiction peut se concevoir comme une perte de contrôle sur un comportement initialement inducteur de plaisir. II peut s'agir de comportements sexuels mais aussi de consommations d'alcool ou d'autres drogues, d'ingestions d'aliments gras et sucrés, de jeux de hasard, de jeux en ligne, d'activités sportives même ou, encore, d'échanges sur les réseaux sociaux - la liste n'est pas limitative. La personne en proie à une addiction se sent poussée à émettre de tels comportements en dépit de leurs conséquences pourtant perçues comme nuisibles: conflits relationnels, problèmes de santé, pertes de temps, d'argent, torts causés à autrui, problèmes judiciaires et autres difficultés sont bien souvent les redoutables corollaires de passages à l'acte débridés. Cette situation provoque une souffrance dans la mesure où la personne a l'impression de ne pas pouvoir s'abstenir ou se réfréner alors qu'elle est vivement consciente qu'il le faudrait, elle pâtit bel et bien de ses comportements excessifs mais elle ressent tout à la fois une forte pression interne à les produire, s'en empêcher provoque un malaise, une tension difficilement supportable qui indique que, d'essentiellement récréatif qu'il était au début, le comportement est à présent devenu compulsif, il vise avant tout à réduire un inconfort. Ces envies impérieuses et obsédantes de poser un acte malgré une motivation " raisonnable " contraire est habituellement désignée sous le terme craving, un vocable anglo-saxon qui ne connait pas d'équivalent français aussi pointu.

Les dépendances aux substances fournissent le modèle par excellence de ce type de difficulté, c'est en tout cas dans ce domaine qu'elles ont été le plus anciennement étudiées. Considérons par exemple le cas d'une personne qui boit de l'alcool à une fréquence telle qu'elle en vient à menacer sa santé, à détériorer ses relations sociales et à hypothéquer son avenir professionnel. Confrontée à pareilles nuisances, elle décide de réduire drastiquement sa consommation, voire de l'arrêter complètement. S'il y a lieu, elle va recourir à une assistance médicale afin de surmonter les problèmes d'accoutumance physique qui se seraient éventuellement installés. Cependant, une fois l'arrêt décidé et les symptômes de sevrage apaisés, la personne n'en fera pas moins l'expérience d'envies douloureuses de s'enivrer de nouveau - le craving - auxquelles elle devra tant bien que mal essayer de résister afin d'éviter la rechute. Le craving constitue le nœud de ce que l'on nommait autrefois les "dépendances psychologiques", c'est-à-dire les dépendances qui se manifestent au delà du besoin somatique d'une substance, lorsque ce n'est pas ou plus le corps qui réclame sa dose par des symptômes brutaux mais l'esprit qui, de façon peut-être moins spectaculaire, semble en peine de trouver son équilibre en dehors des consommations. Et là, qu'il s'agisse de consommations de drogues, de jeux, de crèmes 
glacées, d'Internet ou de sexe, la dynamique apparaît similaire. Quelle que soit l'activité concernée, le sujet en a perdu le contrôle, l'expérience du craving lui restreignant l'exercice performatif d'une modération. L'expression " dépendance psychologique ", par opposition à "dépendance physique ", n'est néanmoins pas la plus heureuse pour désigner ce phénomène car trop empreinte de connotations dualistes opposant le psychique au physique, on lui préfère actuellement celle d'addiction. Au fond, les manifestations somatiques de sevrage peuvent être considérées comme des complications adventices de certaines addictions.

En 1990, Goodman a proposé de déterminer la présence d'un trouble addictif sur la base des critères cliniques répertoriés dans l'encadré 11.1.

\section{Encadré 11.1.}

\section{Critères définitionnels d'une addiction selon Goodman (1990)}

1. Impossibilité de résister aux impulsions à réaliser un comportement

2. Sensation croissante de tension précédant immédiatement le début de la consommation

3. Plaisir ou soulagement durant sa durée

4. Sensation de perte de contrôle pendant le comportement

5. Au moins 5 des 9 critères suivants :

a. Préoccupation fréquente au sujet du comportement

b. Intensité et durée des épisodes plus importantes que souhaitées à l'origine

c. Tentatives répétées pour réduire, contrôler ou abandonner le comportement.

d. Temps important à préparer les épisodes, à les entreprendre ou à s'en remettre

e. Survenue fréquente des épisodes quand le sujet doit remplir ses obligations professionnelles, sociales ou familiales

f. Activités sociales ou professionnels sacrifiées du fait du comportement.

g. Perpétuation du comportement bien que le sujet sache qu'il cause ou aggrave un problème persistant

h. Tolérance marquée : besoin d'augmenter l'intensité ou la fréquence du comportement pour obtenir l'effet désiré

i. Agitation ou irritabilité quand il est impossible de produire le comportement.

6. Le problème dure depuis plus d'un mois

\section{L'addiction sexuelle}

La plupart des personnes chez qui l'on pose un diagnostic d'alcoolisme, d'héroïnomanie ou de jeu pathologique répondent aux critères énoncés par Goodman (cf. encadré 11.1). Il en va probablement de même chez Robert et chez Michel qui se plaignent de leurs comportements sexuels dans les termes exposés dans les encadrés 11.2 et 11.3 ainsi que chez Serge qui relate la problématique sexuelle décrite dans l'encadré 11.4.

Bien que Robert, Michel et Serge puissent tous les trois se reconnaître comme souffrant d'une addiction sexuelle, on repère des différences notoires entre les situations. Chez Robert et Michel, c'est essentiellement la fréquence du comportement sexuel qui pose problème car, en soi, leurs comportements sexuels sont considérés comme faisant partie du répertoire 
acceptable sinon normal de la plupart des gens. Chacun en pense ce qu'il veut mais se masturber et regarder des pornos ne sont en rien des activités pathologiques, pas plus d'ailleurs que le fait d'avoir des partenaires multiples et/ou de les payer pour leurs prestations. Le problème de Robert et de Michel tient essentiellement à l'ampleur qu'ont prise leurs activités sexuelles, la perte de contrôle se solde chez eux par un dérapage quantitatif. Dans leur cas on parlera d'un trouble de l'hypersexualité. Ce n'est pas tout à fait pareil chez Serge dont c'est le comportement lui-même qui est jugé indésirable. En matière d'exhibition de son sexe à des personnes non-consentantes, la norme de fréquence est plutôt du genre tolérance zéro. Il en va de même pour les activités sexuelles avec des enfants et, en général, pour toutes les activités sexuelles qui impliqueraient la souffrance d'une personne non-consentante. Le craving porte ici sur une façon spécifique de s'exciter sexuellement, une façon considérée comme atypique et problématique. On parlera dans ce cas de troubles paraphiliques. II y a bien sûr également des cas où hypersexualité et paraphilie se mêlent, comme en témoigne l'histoire de Bilal reprise dans l'encadré 11.5. Ce serait même d'après certains la loi du genre, au point que l'on ait parfois préconisé l'appellation paraphilia-related disorders (Kafka, 2007) pour désigner l'ensemble des addictions sexuelles. Dans tous les cas il apparaît difficile au sujet de s'abstenir d'un comportement sexuel ressenti comme indésirable tantôt du point de vue de sa quantité, tantôt du point de vue de sa qualité.

\section{Encadré 11.2.}

Pornographie, masturbation et limites de tolérance

Depuis sa prime adolescence, Robert consacre au moins une heure par jour, souvent deux ou trois, à consulter des documents pornographiques. Cela a commencé avec des revues puis ça s'est poursuivi avec des sites. Généralement il se masturbe pendant ou à l'issue de ses séances mais ce n'est pas automatique. Cette activité n'a jamais empiété sur son travail mais elle commence à peser sur sa vie de couple. Patricia, son épouse depuis 7 ans, se montrait initialement tolérante envers ce comportement, un comportement que Robert ne considérait d'ailleurs nullement comme un problème. Puis, progressivement, Patricia s'en est irritée : «Non seulement ça nous empêche de passer des soirées ensemble mais, même si c'est avec des 'femmes virtuelles', j'ai l'impression que tu me trompes, ce n'est pas valorisant pour moi » lui a-t-elle expliqué en substance. Sensible aux arguments, Robert a tenté d'arrêter mais n'y arrive pas : «Ca me travaille toute la journée, explique-t-il. Dès que Patricia quitte la maison, je fonce sur mon ordinateur comme pour rattraper le temps perdu et, tant le soir que le matin, je reste beaucoup plus longtemps à la salle de bain. Patricia n'est pas dupe évidemment, elle menace de me quitter si je ne change pas. "

\section{Un diagnostic délicat}

La frontière entre le normal et le pathologique n'est pas facile à établir, elle est même assez floue. Dans le domaine de la sexualité - comme d'ailleurs dans d'autres - les comportements humains connaissent naturellement d'importantes variations; l'un a un appétit gargantuesque, l'autre un régime acétique, quant aux goûts de chacun, ils apparaissent toujours un peu bizarres aux yeux de qui ne les partage pas. À partir de quand convient-il de se réfréner? En réalité toute perte de contrôle ne pose pas forcément problème. Prenons par exemple l'histoire de Bilal (encadré 11.5). Bilal aurait pu continuer ses montages photos 
de longues années encore sans en éprouver grand désarroi s'il n'avait à un moment donné perçu sa passion sous l'angle d'une enfreinte à ses valeurs: liens familiaux et fantaisies érotiques étaient à ses yeux moralement incompatibles. Là s'origine la détresse de Bilal visà-vis de son comportement. Sans détresse y associée, un comportement sexuel donné, fut-il incontrôlé, ne pourrait tout simplement pas être taxé de "trouble ". En définitive, le caractère intempestif $d^{\prime} u n$ comportement ou d'un désir sexuel est avant tout affaire d'appréciation, l'appréciation du sujet lui-même, de son entourage et de la société dans son ensemble. La détresse ressentie par rapport à l'émission d'un comportement - et donc l'identification d'un trouble - ne découle ainsi pas "naturellement " du comportement luimême, elle résulte encore et surtout de la plus ou moins grande tolérance individuelle et sociale pratiquée à l'égard de ce comportement.

\section{Encadré 11.3.}

\section{Drague compulsive}

Michel a 55 ans, il vit en couple depuis 10 ans avec Isabelle, qu'il aime énormément. Ils viennent $\mathrm{d}^{\prime}$ acheter une maison ensemble.

Depuis que l'internet existe, Michel « chasse » sur les sites. Il passe des heures à séduire des femmes via son ordinateur, il les consomme " à la hussarde », puis les " jette » sans remords. Ce qu'il apprécie, $c^{\prime}$ est la conquête, peu importent les qualités plastiques et intellectuelles de ses partenaires.

Isabelle a découvert le pot-aux-roses il y a quelques mois. Elle a envisagé la séparation puis s'est ravisée. Elle souhaite donner sa chance à Michel. Elle éprouve cependant beaucoup de mal à continuer de faire l'amour avec lui. Elle n'a plus du tout envie en tout cas de se prêter aux jeux sado-maso qu'il apprécie, lui, mais qu'elle ne prise guère.

Michel raconte que, enfant, il a surpris sa mère flirtant avec son amant. Celle-ci aurait « acheté » le silence de Michel en lui offrant un skateboard. Peu après, elle a quitté le domicile familial. Michel a vécu ces évènements comme une trahison.

Michel trouve Isabelle extrêmement attirante. II éprouve cependant des difficultés à l'entreprendre. Quand ils font l'amour, c'est généralement à son initiative à elle, et Michel s'excite parfois difficilement, préoccupé qu'il est par sa crainte de ne pas la satisfaire.

Les regards portés sur l'homosexualité masculine par exemple, sur les relations sexuelles entre un homme adulte et une personne péripubère ou sur les relations extraconjugales multiples ne sont pas les mêmes selon que l'on vit en Californie, en Russie, au Soudan ou aux îles Samoa. La désignation d'un trouble sexuel relève largement de la construction sociale, elle renvoie à un processus normatif qui entend étiqueter comme problématique le fonctionnement singulier de celui qui en est porteur. L'opération est délicate et peut prêter à controverse, que ce soit à l'échelle d'un micro groupe social comme une famille par exemple, voire un couple, ou à celle d'un vaste ensemble comme, par exemple, la société occidentale.

On peut apprécier le caractère épineux des enjeux culturels et politiques brassés par la question du diagnostic d'addiction sexuelle en considérant les débats qui ont émaillé l'histoire du DSM (Diagnostic and Statistical Manual of Mental Disorders), le manuel diagnostic de l'Association psychiatrique américaine qui sert de référence internationale. Avant 1973, I'homosexualité y était cataloguée comme un " trouble ", elle n'a été évacuée de la liste des problèmes de santé mentale qu'à l'issue d'un vote. En 2013, lors de la cinquième édition du DSM, l'étiquette diagnostique " paraphilie " s'est muée en " trouble 
paraphilique ", I'intention était de souligner que les pratiques et intérêts sexuels atypiques, qui définissent les paraphilies, ne doivent pas en soi être considérées comme pathologiques: ils ne constituent un trouble que s'ils occasionnent une détresse et une perturbation significatives du fonctionnement social de la personne, la chose ne coulait manifestement pas de source auparavant. Ces précisions n'ont toutefois pas empêché certains de reprocher à la définition actuelle du trouble paraphilique d'être encore trop encline à asservir le raisonnement clinique à des conceptions morales (Fekhi-Romdhane \& Ridha, 2017 ; Moser \& Kleinplatz, 2005). Par ailleurs, durant la première décennie des années 2000, plusieurs auteurs (Kafka, 2010; Kingston \& Firestone, 2008; Reid et al., 2012) ont milité pour la reconnaissance d'un trouble dit de l'hypersexualité pour désigner des pratiques sexuelles addictives de type normophilique (non paraphilique) - comme dans les cas de Robert et de Michel. L'Association psychiatrique américaine a prudemment préféré rejeter cette proposition, au moins temporairement, arguant d'une insuffisance de preuves à son appui et d'un manque de consensus dans la communauté scientifique (APA, 2013 ; Kor et al., 2013). Sensibles à la subjectivité de la notion d'excès, certains craignaient en effet qu'un tel diagnostic n'aboutisse qu'à stigmatiser des comportements non conformes aux standards dominants, d'autres soulignaient le caractère puissant de la pulsion sexuelle - dont dépend la perpétuation de l'espèce - et, voyant l'obsession, voire la compulsion sexuelle comme un tropisme adaptatif, dénonçaient le danger de médicaliser des manques de retenue qui, au fond, ne relèvent que de l'inconduite (Frances, 2013; Halpern, 2011 ; Levine, 2010 ; Ley, 2012.)

\section{Encadré 11.4. \\ Exhibitionnisme}

Serge a des démêlés avec la justice. Il a exhibé son sexe en érection à une jeune fille qui, pleine de présence d'esprit, a réussi à photographier Serge avec son smartphone et a ensuite porté plainte. C'est la honte. Suite aux faits, Nahima, sa compagne, a quitté Serge non sans en ébruiter la raison.

Ce n'est pas la première fois que Serge s'exhibe de la sorte. D'habitude il récolte des réactions effarouchées, une situation qui l'excite énormément et tend à motiver une répétition de l'acte en dépit d'une conscience pourtant claire du caractère peu glorieux et illégal de celui-ci. " C'est plus fort que moi » explique-t-il. Cela se produit souvent à la suite d'une frustration ou d'une dispute. "J'entre alors dans une sorte de transe ", un état émotionnel intense où rage et excitation sexuelle s'entremêlent et où des actes sexuels "méchants" comme l'éjaculation faciale ou l'exhibition deviennent des idées fixes. "La plupart du temps je parviens à évacuer mes tensions, par exemple en me masturbant, mais parfois ça dérape. " Le risque de dérapage est en l'occurrence plus important lorsqu'un état d'esprit de ce genre s'empare de Serge alors qu'il traîne en ville tard le soir sous l'emprise de l'alcool ou du cannabis.

Ca n'allait pas fort dans la vie de Serge ces derniers temps. Sa relation avec Nahima allait à veau l'eau, ils se disputaient tout le temps. En plus il venait de perdre son emploi de barman et avait été contraint $d^{\prime}$ 'emprunter à sa mère de quoi faire la soudure, une mère qu'il avait toujours perçue comme un personnage autoritaire, prompt à le critiquer. 
Encadré 11.5.

Cyberaddiction sexuelle

Bilal, 35 ans, passe des journées entières à télécharger des images pornographiques et à les monter en des récits pour lui excitants. La thématique tourne essentiellement autour de femmes dominatrices. Avec Photoshop, il colle parfois les visages de certaines de ses connaissances sur les corps des actrices porno. Il consacre beaucoup de temps à cette activité. Ca ne l'empêche pas de travailler mais ne parlons pas de faire du sport, de rencontrer des amis, ou d'activités culturelles. Dernièrement, il s'est surpris à coller sur des corps d'actrices porno le visage de ses nièces. Ce fut le déclic. « Ce n'est plus normal, il faut que j'arrête ça. ॥

Bilal vit seul. II désespère de pouvoir un jour vivre en couple. II a bien eu quelques copines, mais jamais de relations pénétratives réussies. Ça le stresse bien trop et, du reste, les relations "normales » lui semblent tellement moins excitantes que ses fantasmes masochiques.

Bilal s'interroge. "Mon addiction me sert de refuge. Mais cette addiction ne fait-elle pas que renforcer mon inaptitude aux relations 'normales' ?"

\section{Ampleur de la problématique}

Étant donné l'incertitude nosologique qui entoure la notion d'addiction sexuelle, il apparaît difficile d'évaluer l'ampleur de la problématique. Dans une cohorte néo-zélandaise, Skegg et al. (2010) ont observé que $13 \%$ des hommes et $7 \%$ des femmes déclaraient avoir éprouvé un défaut de contrôle sur leurs besoins ou comportements sexuels durant I 'année écoulée. Ils n'étaient toutefois que 1 à 2,8 \% à trouver que cela avait eu un impact négatif sur leur vie. En partant de certaines statistiques de demandes d'aide, une société américaine de promotion de la santé sexuelle (SASH, 2008) a estimé à environ 3 à $5 \%$ la part de la population présentant un problème de compulsion sexuelle (source citée par Tripodi et al., 2012.) On estime que la difficulté touche davantage les hommes que les femmes et davantage les gays que les hétéros (Black, 2000 ; Kafka, 2010). Avec le développement des pratiques de sexe en ligne, on a en tout cas assisté au cours des dernières années à une véritable explosion des problèmes de dépendance ciblant l'une ou l'autre forme de cybersexualité (Carnes et al., 2007.) II est vrai que l'usage d'internet rend toute une série d'activités sexuelles à la fois accessibles et abordables comme jamais auparavant, le tout dans des conditions d'anonymat qui leur confèrent un pouvoir d'attraction extraordinaire. L'internet bénéficie, selon l'expression de Cooper (1998), d'une condition "triple A» (accessible - abordable anonyme) qui en fait un médium addictif puissant.

\section{Place du conjoint dans l'abord du problème}

La question du diagnostic ne fait pas l'objet d'un consensus ferme, c'est le moins qu'on puisse dire. Elle est le lieu d'une tension entre deux mouvements contradictoires : convient-il de rapporter la souffrance suscitée par un comportement sexuel donné plutôt aux excès de son auteur ou plutôt à des jugements excessifs concernant ce comportement ? Quelle est la juste mesure ? Ce n'est pas une simple question de rhétorique, c'est la question qui se pose à tout individu, à tout couple qui consulte pour un comportement sexuel problématique ; la 
manière d'y répondre va déterminer la ou les mesure(s) à adopter afin de réduire la souffrance.

Pour un couple en consultation, l'étiquetage de la difficulté représente un enjeu de pouvoir : qui est le "malade ", qui doit changer et qui est la "victime ", qui est dans son droit ? Le thérapeute peut se voir interpelé à ce sujet comme on interpellerait un arbitre dépositaire d'un savoir normatif. Le diagnostic devient dès lors une opération qu'il convient de mener avec prudence, elle ne peut se résumer à n'être qu'un vocable posé sur un comportement. Dans le cas de Marc et Mireille par exemple (tableau 6) il ne semble ni plus ni moins sensé de dire de Marc qu'il présente un problème addictif voire paraphilique que de dire de Mireille qu'elle souffre d'un manque de confiance en elle voire de psychorigidité, chacun de ces diagnostics peut se justifier et, en même temps, apparaitre extrêmement réducteur. Ce qui importe c'est de rapporter la souffrance à la situation singulière du couple, de prêter attention à la multiplicité de ses tenants et de questionner les possibilités de changement à ces multiples niveaux.

\section{Encadré 11.6.}

\section{Une pratique particulière, des questions déstabilisantes}

Mireille était effondrée. En nettoyant les armoires, elle avait trouvé un " godemiché » que Marc, son compagnon, dissimulait dans son tiroir à chaussettes. Il s'agissait d'un plug anal en réalité. Marc avait découvert quelques années auparavant le plaisir intense que lui procurait une stimulation prostatique à l'aide de cet instrument tandis qu'il se masturbait, il répétait depuis lors régulièrement l'expérience, au moins deux fois par semaine, lorsque Mireille n'était pas à la maison. Ces explications ne rassuraient aucunement Mireille : Pourquoi Marc utilisait-il ce truc aussi souvent et ne lui faisait-il plus l'amour qu'une fois par mois au grand maximum ? Pourquoi lui avait-il caché cette pratique ? Pourquoi même avait-il eu l'idée d'y recourir ? "Tous les hommes ne font pas ça tout de même, disait-elle, la sodomie c'est un plaisir de gay, non ? " Elle se sentait niée dans sa féminité et parlait de rupture, il lui était inimaginable de continuer à vivre avec un " pervers".

En cas de reconnaissance d'une dynamique addictive, une des visées essentielles du traitement consiste à réduire voire arrêter totalement le comportement sexuel problématique. Autrement dit c'est sur l'individu porteur du symptôme addictif que se focalise l'intention thérapeutique. C'est lui, plutôt que le couple dans son ensemble qui se voit désigné comme le patient, le client ou, disons, le « premier bénéficiaire » du traitement. Ceci ne signifie évidemment pas qu'il faille exclure le partenaire du processus thérapeutique, au contraire, le patient en viendra presqu'inéluctablement à interroger la dimension conjugale susceptible de participer à son problème et/ou à sa résolution, dès lors la participation du partenaire à une réflexion conjointe et aux dispositifs de changement peut souvent s'avérer cruciale. Cependant, même s'il faut reconnaitre avec Goodman (1998) que la dynamique conjugale contribue fréquemment à l'évolution d'une addiction sexuelle, il demeure exceptionnel que celle-ci repose exclusivement sur des facteurs liés au partenaire. Du coup, même si l'on pousse le curseur fort loin en matière d'implication du conjoint, par exemple en incluant systématiquement ce dernier lors de chaque séance, les objectifs 
thérapeutiques ne seront jamais parfaitement équilibrés. Conceptuellement, nous nous situerons toujours dans le champ d'une thérapie individuelle avec participation plus ou moins importante du partenaire plutôt que dans le domaine de la thérapie de couple stricto sensu. Dans le traitement des dépendances sexuelles, les recommandations qui font autorité s'accordent souvent à considérer le travail avec le conjoint comme souhaitable, jamais comme indispensable (Birchard, 2015 ; Swisher, 1995 ; Tripodi et al, 2012.)

\section{Traitement}

\section{Principes généraux}

Le traitement d'une addiction a pour principe général de considérer le comportement indésirable comme une réponse à des situations stressantes, une réponse certes inadaptée mais relativement efficace. Le stress ressenti peut avoir des origines diverses mais sa nature n'est pas toujours très claire pour le sujet lui-même, lequel, pétri d'une dynamique de passage à l'acte, le perçoit avant tout comme une expérience de craving, comme une envie obsédante de poser son comportement addictif. Ceci requiert en premier lieu une démarche exploratoire: il s'agit d'une part d'interroger les circonstances externes et internes qui président ou présidaient habituellement au comportement problème et, d'autre part, d'interroger le plaisir manquant qui résulte de l'abstention de ce même comportement. II conviendra ensuite d'élaborer d'autres modalités de réponse au stress.

Ce type d'approche a été systématisé dans les deux grands modèles de référence qui structurent la prise en charge cognitivo-comportementale des problèmes d'addiction. Le premier, dénommé Prévention de la rechute, a été initialement élaboré par Marlatt et Gordon (1985) dans le domaine des dépendances aux substances, il s'est par la suite élargi à l'ensemble des problèmes addictifs, aux addictions sexuelles notamment sous l'impulsion d'auteurs comme Hall (2013), Laws (2003) et Marshall et al. (1990, 1999). Ce modèle insiste sur la nécessité d'une analyse fine des circonstances à risques de rechute, sur leurs enchainements et leurs interrelations parfois complexes afin de restituer au sujet la possibilité d'en prévenir l'occurrence ou d'en déjouer la logique funeste (Kempeneers, 1999.) Le second modèle dit Motivationnel a été proposé par Miller et Rollnick en 1991 (2012 pour la traduction française de la deuxième édition.) Inspiré des travaux de Prochaska et DiClemente sur les processus de changement dans l'émission de comportements volontaires, (Prochaska \& DiClemente, 1982 ; Prochaska, Norcross \& DiClemente, 1994), ce modèle se centre sur l'ambivalence du sujet dépendant, un sujet partagé entre, d'une part, son aspiration à se déprendre de ses comportements problématiques et, d'autre part, les agréments que ceux-ci lui apportent ainsi que les désagréments qu'entraînerait l'abstention. L'accent est placé sur la reconnaissance explicite des facteurs de résistance au changement et sur le soutien du patient dans un parcours jalonné d'hésitations, d'avancées et de reculs. La motivation au changement est ici perçue comme un processus dynamique qu'il convient pour le thérapeute d'influencer adéquatement en fonction de l'évolution particulière du patient. 
Prévention de la rechute et approche motivationnelle forment l'épine dorsale conceptuelle de la prise en charge cognitivo-comportementale des addictions. Nous pouvons nous représenter leur jeu à partir de la figure 11.1 qui résume la problématique d'une personne en proie à une addiction sexuelle. La prévention de la rechute se centre sur une analyse détaillée des facteurs de stress inhérents à l'abstention du comportement problématique ainsi que sur les moyens de leur réduction. L'approche motivationnelle, elle, a pour principal dessein d'éclairer la dynamique dans son ensemble et de proposer des interventions visant tantôt à réduire les conséquences aversives de l'abstention - des interventions de type prévention de la rechute notamment -, tantôt à majorer les bénéfices de la cessation, ce qui, comme nous le verrons plus loin, ne se résume pas forcément à la seule réduction de ses inconvénients. Le cas échéant, lorsque la personne se sent incapable de réduire son comportement addictif, il pourra aussi s'agir d'explorer les moyens de limiter les conséquences négatives de son maintien. C'est là par exemple que le sujet pourra envisager de se séparer de son conjoint afin de diminuer la souffrance relationnelle, ou pour en trouver un autre plus " tolérant". C'est là aussi qu'il conviendra peut-être de se départir d'une certaine vision conventionnelle de la "normalité ", ou de s'interroger sur de possibles dispositifs sanitaires permettant de limiter l'exposition aux infections sexuellement transmissibles (IST). Pareilles interventions destinées à réduire les problèmes occasionnés par un comportement addictif maintenu inchangé sont quelquefois désignées comme relevant de la réduction des dommages, mais c'est surtout le cas lorsqu'elles émanent de politiques publiques; on songe, dans le cas des problèmes de dépendances aux substances par exemple, à des programmes d'échanges de seringues, à la légalisation de certains produits psychotropes ou à d'autres mesures du même tonneau.

Ces modèles généraux permettent de conceptualiser l'intervention dans le champ des addictions, ils lui sont assez spécifiques. Cependant, selon les cas particuliers, l'intervention se devra d'intégrer aussi des approches non spécifiques à la problématique addictive. Le stress auquel le sujet a tendance à réagir par des comportements addictifs devrait pour bien faire être géré différemment. En fonction des difficultés particulières de la personne, diverses approches seront conviées. Affirmation de soi par exemple, thérapie beckienne, activation comportementale, méditation en pleine conscience ou, encore, résolution de problème sont autant de techniques applicables au cas par cas; la thérapie de couple et la sexothérapie entrent également dans cette catégorie.

Les interventions non spécifiques se conçoivent habituellement comme un travail sur les " comorbidités", sur les difficultés associées à l'addiction. Ceci soulève la question d'éventuelles comorbidités propres à l'addiction sexuelle. II est communément admis que la palette entière des psychopathologies se trouve surreprésentée dans le champ général des addictions, ce qui est fort logique puisque les conduites addictives servent de moyen de régulation émotionnelle. Quant à savoir si les addictions sexuelles s'accompagnent de difficultés caractéristiques qui les distingueraient des autres addictions, la question reste ouverte. Certains auteurs y ont discerné un lien privilégié avec des troubles précoces de l'attachement (Black, 2000 ; Poudat, 2005), avec des problèmes de fragilité narcissique et avec des dysfonctions sexuelles (Birchard, 2015) mais, dans l'état actuel des choses, ceci relève de l'impression clinique davantage que de l'observation statistique. Des difficultés de 
ce type transparaissent en tout cas assez clairement dans les histoires de Michel, de Robert, de Serge et de Bilal (encadrés 11.2 à 11.5).

Figure 11.1. Le processus addictif : ambivalence du sujet dépendant vis-à-vis de son comportement problème (adapté de Kempeneers, 1999)

Conséquences pénibles de la production excessive du comportement sexuel

Exemples : conflits interpersonnels, honte, IST, pertes de temps,

d'argent...

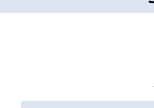

Motivation à l'arrêt

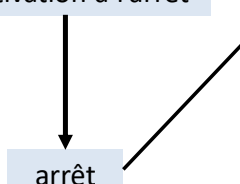

l'abstention

(stress ?manque de plaisir?)

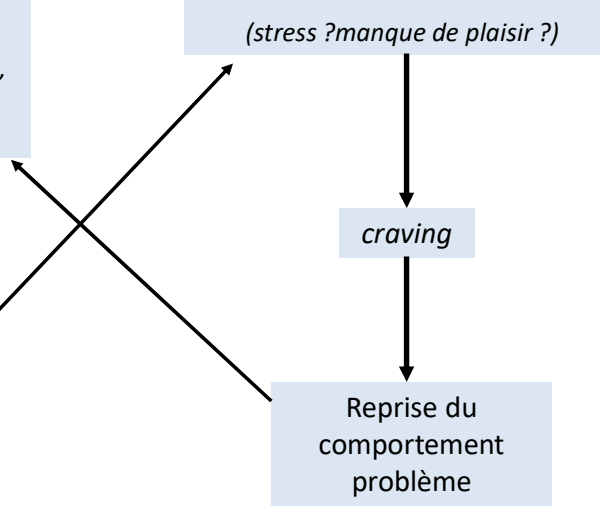

\section{La révélation du problème}

La découverte par le partenaire du comportement sexuel supposément addictif de son conjoint peut être vécue comme un véritable traumatisme. Une ligne rouge a été franchie ou est sur le point de l'être, les choses ne sont soudain plus comme le partenaire croyait qu'elles étaient, ses repères, la vision qu'il avait de lui-même, de son conjoint et de son couple, tout cela s'en trouve bouleversé. Le couple est remis en question et c'est d'ailleurs bien souvent ce point qui constitue le premier motif de consultation : "Je ne peux pas tolérer ça. Si ce n'est pas simple pour toi de t'en abstenir, c'est que tu es malade, alors fais-toi soigner. C'est à cette condition seulement que nous pourrons poursuivre ou recommencer notre relation " entend-on régulièrement en substance. Se pose alors la question du diagnostic, laquelle va déterminer les principaux axes du traitement.

Le traumatisme déclenché par le dévoilement du problème peut requérir une prise en charge spécifique du partenaire, de préférence par un thérapeute autre que celui consulté pour le trouble addictif. Dans tous les cas, les deux partenaires devront tenir compte d'éventuelles manifestations symptomatiques associées au stress post-traumatique : abattement, tristesse, réactions agressives et évitements ont effectivement quelque probabilité de se manifester chez le conjoint. La reconnaissance de telles manifestations, leur acceptation et, idéalement, leur gestion adaptée aideront à éviter que le climat relationnel ne se dégrade davantage encore. Promouvoir l'empathie réciproque au sein le couple est souvent indispensable. 
Tripodi et ses collaboratrices (2012) vont jusqu'à préconiser aux patients addicts de dévoiler eux-mêmes, si ce n'est déjà fait, leur problème à leur conjoint. L'argument, emprunté à Bird (2006), est que, par delà les secousses que la révélation provoque inévitablement dans la relation, la transparence favoriserait à terme une meilleure évolution aussi bien du couple que du patient lui-même. Il vaut tout de même mieux y réfléchir, en l'occurrence peut-être avec un thérapeute en qui l'on a confiance, d'où l'importance d'un cadre de thérapie individuelle.

\section{Zones blanches et lignes rouges}

Le patient addict sera assez rapidement invité à installer des "zones blanches ", c'est à dire des périodes de temps durant lesquelles il tâchera, quoi qu'il lui en coute, de s'abstenir de ses comportements sexuels problématiques. Ces zones blanches ont plusieurs visées. Elles ont d'abord un but exploratoire ; tenir n'est pas forcément évident et il s'agit pour le patient d'examiner en quoi l'exercice peut s'avérer difficile. Existe-t-il à cet égard des circonstances plus critiques que d'autres? Des moments sensibles (par exemple le soir, quand je suis seul ?), des endroits à risques (le bureau où se trouve mon ordinateur, le quartier de la gare par lequel je passe après mon travail ?) ou encore des états d'esprits problématiques (lorsque je me sens frustré, lorsque je m'ennuie ?) A contrario certaines situations peuvent se révéler protectrices. La présence de certaines personnes par exemple, certaines activités, certains lieux ou certains états d'esprit peuvent rendre l'expérience du craving moins probable, moins intense ou bien contribuer à ce qu'il soit moins difficile y résister.

L'établissement des zones blanches dépend de plusieurs facteurs. Les lignes rouges décrétées par le conjoint en font évidemment partie. Pour Michel, c'est clair, il convient de s'interdire toute relation extraconjugale. Pour Serge aussi c'est clair, il s'agit de s'abstenir de toute exhibition, à cette nuance près que c'est ici la loi et non le conjoint qui fixe la ligne rouge. Pour Robert, c'est un peu plus délicat : toute masturbation, tout visionnage de porno doit-il être exclu ? Probablement pas. L'objectif serait pour lui de limiter plutôt que de supprimer un comportement dont c'est essentiellement l'ampleur qui déplait à la compagne. Le niveau de limitation doit être discuté avec elle. L'établissement des zones blanches doit également tenir compte de la dynamique particulière de l'acte problématique.

Charles, par exemple, pouvait distinguer chez lui deux formes de masturbation: l'une, jugée acceptable, se produisait le soir, au lit, l'autre, identifiée comme compulsive, survenait en journée en réaction à des ruminations dysphoriques. Seule la seconde forme était jugée indésirable.

Les zones blanches doivent encore être établies dans les limites du supportable. Un « plus jamais " motivé par une haute ambition peut avoir un effet pervers décourageant, mieux vaut s'en tenir pour commencer à des durées plus raisonnables : s'abstenir pendant un mois ou une semaine, par exemple, ou trois jours par semaine, ou six heures par jour sont des objectifs certes plus modestes mais sans doute plus réalistes.

Outre des zones d'exploration, les zones blanches sont également des terrains d'exercice. Leur seconde visée consiste effectivement à expérimenter des ressources permettant de 
gérer les circonstances à risques autrement que par la consommation sexuelle. Ainsi Michel a-t-il décidé de troquer son smartphone pour un téléphone cellulaire ancien modèle sans accès internet. II a également confié son ordinateur portable à Isabelle qui le conservait sous clé dans son bureau, sauf à la demande expresse du patient. Michel pensait que ces dispositions raréfieraient le craving qu'il ressentait fréquemment lorsqu'il était seul face à un écran avec la possibilité de surfer sans contrôle sur les sites de rencontre. Au fil du temps, il $s^{\prime}$ est mis aussi à requalifier ses expériences de craving en y détectant des difficultés plus fondamentales. Il se disait "Ce n'est pas tant que j'ai envie de sexe, le sexe n'est au fond qu'une mauvaise réponse à un problème de sensibilité personnelle: dès que je suis seul et désceuvré, j'ai tendance à me sentir mal, triste, peu aimable et je rumine des impressions de rejet. Ne pourrais-je gérer cela autrement qu'en me distrayant dans la drague, dans l'illusion qu'elle me donne d'être aimé de quelqu'un ? "Et, de fait, Michel s'est mis à la tâche, il essayait tantôt de trouver d'autres dérivatifs à son malaise (sport, musique, bricolage), tantôt de relativiser celui-ci en objectivant ses solides liens d'affection avec Isabelle, ses enfants, ses amis. Sensibilisée à la détresse de Michel, Isabelle s'est de son côté appliquée à multiplier les petits gestes affectueux. Les conjoints se sont encore interrogés ensemble sur la sexualité au sein de leur couple, ils ont élargi la palette de leurs comportements érotiques et en ont tiré de nouvelles satisfactions. Toutes ces mesures rendaient plus facile de s'abstenir.

Les zones blanches ont enfin une mission de renforcement. Elles permettent aux personnes d'intégrer qu'il est possible de se déprendre d'un comportement sexuel problématique, ne serait-ce que durant une période limitée. Progressivement, le sujet gagne de la confiance dans les ressources qu'il parvient à déployer pour améliorer la qualité de sa vie abstinente, son sentiment d'auto-efficacité s'accroît au fil du traitement de sorte que, c'est le but, il devient envisageable d'élargir au maximum les périodes de modération.

\section{Tableau décisionnel}

II n'est pas inutile de proposer à la personne de transcrire quelques-unes de ses réflexions sur un tableau synoptique dit décisionnel. Préconisé par Miller et Rollnick (2013), l'exercice consiste à répertorier dans un tableau à quatre cases les avantages et les inconvénients liés d'une part à l'émission du comportement problématique, d'autre part à l'abstention. Ceci permet d'identifier et de conserver à l'esprit les éléments de motivation susceptibles de faire pencher la balance tantôt du côté du passage à l'acte tantôt du côté de la modération. Attendu que c'est bien la modération qui est recherchée, les efforts de changement pourront cibler spécifiquement des éléments susceptibles de contribuer à ce résultat. À titre d'exemple, on trouvera le tableau décisionnel (tableau 11.1) rempli par Robert. 
Tableau 11.1. Exemple de tableau décisionnel

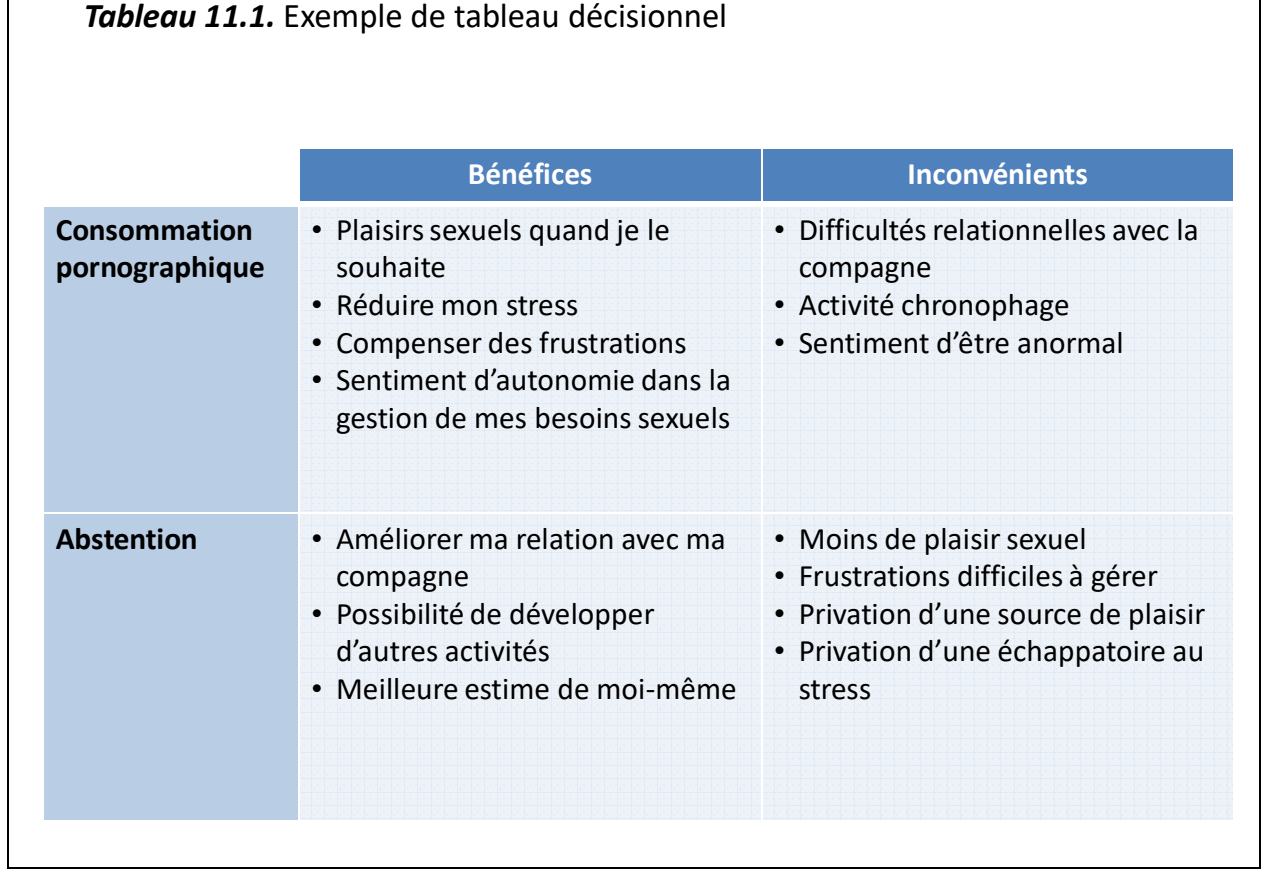

La case dédiée aux bénéfices du comportement sexuel problématique répertorie les multiples renforcements de celui-ci, il s'agit d'en reconnaître l'existence et d'instiller l'idée que, si l'obtention de certains de ces avantages peut paraître légitime, d'autres moyens que le comportement problématique peuvent aussi être déployés à cette fin. On procède en somme ici à une analyse fonctionnelle classique de la difficulté.

La case réservée aux inconvénients du comportement addictif met en lumière les motivations " négatives " au changement, celles qui font souffrir le sujet et le mènent à rêver à l'arrêt. Sur le plan thérapeutique, il ne sert à rien de surenchérir à ce niveau, les conséquences aversives du comportement précisément jugé "problématique » sont en général bien connues du patient mais elles ne suffisent pas à garantir sa cessation, c'est là la nature même de l'addiction. Cette case peut par ailleurs guider les interventions visant à réduire les dommages. De fait, lorsque l'abandon du comportement addictif apparaît impossible ou trop difficile, la ressource demeure toujours d'œuvrer à la réduction des inconvénients qui lui sont associés. Ainsi Robert s'est-il un moment interrogé sur la nécessité ou non de maintenir sa relation avec Patricia, il s'est questionné sur l'importance relative $d^{\prime}$ 'une relation conjugale en matière $d^{\prime}$ accomplissement existentiel et sur la relativité de la notion de normalité. II valait probablement mieux que de telles réflexions se verbalisent en I'absence de Patricia. Quoi qu'il en soit, au terme de cette clarification, Robert a choisi de renouveler son engagement auprès de sa compagne. La perspective d'améliorer sa qualité de vie tout en observant une modération sexuelle l'y a beaucoup aidé.

C'est habituellement en remplissant la case des inconvénients de l'abstention, et surtout en la développant, que se précisent les principales cibles de l'intervention. Suite à un examen des circonstances dans lesquelles il éprouvait des difficultés à se modérer, Robert s'est perçu comme un personnage plutôt démuni en matière de gestion émotionnelle, il a notamment vu dans ce problème l'expression d'une tendance à s'autocritiquer et à projeter ses 
sempiternelles autocritiques dans le chef de ses proches. Un sentiment diffus d'insuffisance personnelle l'accompagnait en quasi permanence et le rendait plutôt crispé dans les relations, ceci n'était pas sans lien d'ailleurs avec une forme larvée d'anxiété sexuelle qui l'empêchait de se laisser paisiblement aller dans l'intimité avec Patricia. Robert était finalement fort exigeant avec lui-même, inquiet de démériter. Mais ses exigences étaientelles bien réalistes ? N'était-il pas possible pour Robert de voir les choses différemment, de s'essayer à d'autres rapports avec les gens que ceux dictés par ses complexes ? Robert a pris également la mesure du peu de plaisir qu'il prenait dans l'existence en dehors des activités sexuelles, ce qui peut-être contribuait à faire de lui un véritable obsédé. N'était-il pas possible d'explorer d'autres activités sources de bien-être ? Sans entrer davantage dans le détail du cheminement de Robert, notons que c'est essentiellement à ce stade du traitement qu'interviennent de multiples techniques thérapeutiques non spécifiques au traitement des addictions, en ce compris les thérapies de couple et les thérapies sexuelles. Pointer les inconvénients de la modération revient à pointer les comorbidités. Toutes ces difficultés auxquelles les conduites addictives permettaient d'échapper momentanément méritent probablement des réponses plus nuancées.

La dernière case, celle des bénéfices de l'abstention, permet au patient de visualiser ce qu'il gagne au changement. Au départ, l'aspiration au changement est essentiellement motivée par le désir de s'affranchir des désagréments produits du comportement addictif, il est toutefois possible d'aller plus loin dans la perception des avantages de l'abstention de manière à rendre celle-ci plus désirable encore. Pour Robert par exemple, l'amélioration de sa relation avec Patricia ne s'est pas bornée à éviter simplement les conflits, le traitement a également visé à optimiser la satisfaction sexuelle au sein du couple. De même, la récupération d'un temps ordinairement alloué aux conduites addictives a permis à Robert d'expérimenter de nouvelles activités plaisantes: lecture, cinéma, photographie, sport en salle et sorties en couple se sont révélés d'excellents renforcements.

\section{Conclusions}

Les excès sexuels d'un des partenaires peuvent poser problème au sein d'un couple. Ils ne relèvent pas forcément d'une problématique addictive et il convient d'aborder la question du diagnostic avec circonspection. Lorsqu'un problème d'addiction sexuelle est effectivement reconnu, il est préférable que le traitement de celle-ci s'opère dans le cadre d'une thérapie individuelle plutôt que dans celui d'une thérapie de couple. Pour autant, le traitement de l'addiction ne manquera pas d'interroger la dynamique conjugale, que ce soit en tant contribution causale, de facteur d'amélioration ou d'élément à améliorer. La question du maintien du couple et de son coût se pose régulièrement, elle mérite d'être abordée par chaque partenaire séparément, la configuration dans laquelle chaque conjoint a son propre thérapeute est dès lors assez fréquente. En cas de poursuite du couple, la mise en commun de certaines réflexions peut s'avérer nécessaire. II faut en effet négocier les limites d'un comportement sexuel acceptable pour chacun. Le cas échéant, le conjoint peut en outre fournir un soutien précieux aux changements qu'essaye d'apporter le patient addict dans son propre fonctionnement. Le fonctionnement du couple peut également devenir lui-même 
l'objet d'un changement, notamment au niveau sexuel, ce qui implique la participation active du conjoint. Dans ces conditions, des techniques de thérapie de couple et de sexothérapie sont souvent indiquées.

\section{Références}

Birchard, T. (2015). CBT for compulsive sexual behaviour: a guide for professionals. London, UK: Routledge.

Bird, M.H. (2006). Sexual addiction and marriage and family therapy: facilitating individual and relationship healing through couple therapy. Journal of Marital and Family Therapy, 32, 297-311.

Black, D.W. (2000). The epidemiology and phenomenology of compulsive sexual behavior. CNS Spectrums, 5(1), 26-72.

Carnes, P., Delmonico, D.L., Griffin, E., \& Moriarity, J.M. (2007). In the shadow of the net. Breaking free of compulsive online sexual behavior $-2^{\text {nd }}$ edition. Center City, MN: Hazelden.

Cooper, A. (1998). Sexuality and the internet: surfing into the new millennium. CyberPsychology and Behavior, 1, 181-187.

Fekhi-Romdhane, F., \& Ridha, R. (2017). Les troubles paraphiliques dans le DSM-5 : analyse critique et considérations médicolégales. Annales Médico-psychologiques, doi: 10.1016/j.amp.2017.03.022

Frances, A. (2013). Sommes-nous tous des malades mentaux ? La vérité sur le DSM-5. Paris, F : Odile Jacob

Goodman, A. (1990). Addiction: definition and implications. British Journal of Addictions, 85, $1403-8$

Goodman, A. (1998). Sexual addiction: An integrated approach. Madison, CT: International Universities Press.

Hall, P. (2013). Understanding and treating sex addiction. Hove, UK: Routledge.

Halpern, A.L. (2011). The proposed diagnosis of hypersexual disorder for inclusion in DSM-5: unnecessary and harmful. Archives of Sexual Behavior, 40(3), 487-8.

Kafka, M.P. (2007). Paraphilia-related disorders. In S.L. Leiblum (Ed.), Principles and practice of sex therapy (4th ed.). New York, NY: Guilford

Kafka, M.P. (2010) Hypersexual disorder: a proposed diagnosis for DSM-V. Archives of Sexual Behavior, 39(2), 377-400

Kingstone, D.A., \& Firestone, P. (2008). Problematic hypersexuality: a review of conceptualisation and diagnosis. Sexual Addiction and Compulsivity, 15(4), 284-310. 
Kor, A., Fogel, Y., Reid, R.C., \& Potenza, M.N. (2013). Should hypersexual disorder be classified as an addiction? Sexual Addiction and Compulsivity, 20(1-2), doi: 10.1080/10720162.2013.768132.

Laws, D.R. (2003). The rise and fall of Relapse prevention. Australian Psychologist, 38(1), 2230.

Levine, S.B. (2010). What is sexual addiction? Journal of Sex and Marital Therapy, 36, 261275. doi: 10.1080/00926231003719681.

Ley, D. (2012). The myth of sex addiction. Lanham, MD: Rowman and Littlefield

Marlatt, G.A. \& Gordon, J.R. (1985). Relapse prevention: maintenance stategies in the treatment of addictive behaviors. New York, NY: Guilford Press

Marshall, W.L., Anderson, D., \& Fernandez, Y. (1999). Cognitive behavioural treatment of sexual offenders. Chichester, UK: Wiley and sons.

Marshall, W.L., Laws, D.R., \& Barbaree, H.E. (1990). Handbook of sexual assault. New York, NY: Guilford.

Miller, W.R. \& Rollnick, S. (1991). Motivational Interviewing: preaparing people to change addictive behavior. New York, NY: Guilford

Miller, W.R. \& Rollnick, S. (2013). L'entretien motivationel : Aider la personne à engager le changement, $2^{\text {ème }}$ éd. Paris, F : InterEditions.

Mozer, C., \& Kleinplatz, P.J. (2005). DSM-IV-TR and the paraphilia : an argument for removal. Journal of Psychology and Human Sexuality, 17, 91-109.

Prochaska, J.O., \& DiClemente, C.C. (1982). Transtheorical therapy: toward an integrative model of change. Psychotherapy: Theory, Research and Practice, 19(3), 276-88.

Prochaska, J.O., Norcross, J.C., \& DiClemente C.C (1994). Changing for Good, New York, NY: Avon Books, Inc

Poudat, F-X. (2005). La dépendance amoureuse. Paris, F : Odile Jacob.

Reid, R.C., Carpenter, B.N., Hook, J.N., Garos, S., Manning, J.C., Gililand, R.,... Fong, T. (2012). Report of findings in a DSM-5 field trial for hypersexual disorder. Journal of Sexual Medicine, 9, 2868-2877. doi: 10.1111/j.1743-6109.2012.02936.x.

SASH - Society for Advancement of Sexual Health. (2008). Sexual addiction. Retrieved September 21, 2008, from http://www.sash.net/

Skegg, K., Nada-Raja, S., Dickson, N., \& Paul, C. (2010). Percieved "Out of Control" sexual behavior in a cohort of young adults from the Dunedin Multidisciplinary Health and Development Study. Archives of Sexual Behavior, 39, 968-978.

Swisher, S. (1995). Therapeutic interventions recommended for treatment of sexual addiction/compulsivity. Sexual Addiction and Compulsivity, 2, 31-39. 
Tripodi, F., Giuliani, M., Petruccelli, I., Simonelli, C. (2012). Sexual addiction: theory, causes and therapy. In H. Porst, \& Y. Reisman (Eds.), The European Society of Sexual Medicine syllabus of sexual medicine (pp. 1042-72). Amsterdam, NL: Medix Publishers. 